EPJ manuscript No.

(will be inserted by the editor)

\title{
Medium effects on $\phi$ decays to dilepton and kaon-antikaon pairs in relativistic heavy ion reactions
}

\author{
E. Santini, G. Burau, Amand Fäßler, C. Fuchs \\ Institut für Theoretische Physik, Universität Tübingen, Auf der Morgenstelle 14, D-72076 Tübingen, Germany \\ Received: date / Revised version: date
}

\begin{abstract}
We consider the role of rescattering of secondary kaons on the dilepton branching ratio of the $\phi$ meson. In-medium mass modifications and broadening of kaons and $\phi$ mesons are taken into account. We find in the framework of a Bjorken scenario for the time evolution of the expanding fireball that the $\phi$ yield from dimuons is moderately or at least only slightly enhanced compared to that from kaon-antikaon pairs. The relation to experimental yields measured by the NA49, NA50 and CERES Collaborations at CERN SPS and the PHENIX Collaboration at RHIC is discussed.
\end{abstract}

PACS. 25.75.-q Relativistic heavy ion collisions - 14.40.Aq pi and K mesons - 14.40.Ev phi mesons 13.75.Lb meson-meson interactions

\section{Introduction}

Relativistic heavy ion (RHI) collisions present an unique opportunity to create nuclear matter at high density and temperature where hadron properties become different and the phase transition to quark matter with signature of the deconfinement and restoration of chiral symmetry is expected. Strangeness enhancement in relativistic nucleusnucleus collisions compared to nucleon-nucleon collisions has been suggested as a possible signal for the formation of the quark gluon plasma (QGP) [1]. The dominant production of $s \bar{s}$ pairs via gluon-gluon interaction in the plasma may result in an enhanced number of strange and multistrange particles produced after hadronization. In particular, the production and decay of the $\phi$ meson have been long recognised as an important probe for the state of matter produced in RHI collisions [1,2]. The possible enhancement of its yield has been suggested as an important signature of the formation of a QGP state [2], in which free $s \bar{s}$ pairs would coalesce to form $\phi$ mesons, whereas their production in $p p$ collisions is suppressed according to the Okubo-Zweig-Iizuka rule [3].

Phi meson production was measured in central $\mathrm{Pb}+\mathrm{Pb}$ collisions at $158 \mathrm{~A} \mathrm{GeV}$ at the CERN SPS. The NA49 Collaboration identified the phi meson via the decay channel $\phi \rightarrow K^{+} K^{-}$4, while the NA50 Collaboration identified it via the $\phi \rightarrow \mu^{+} \mu^{-}$decay [5]. It was found that the extracted number of phi mesons from the dimuon channel exceeded those number extracted from the $K^{+} K^{-}$channel by a factor between two and four 6 .

It has been argued that this difference could be at least partially attributed to the fact that not all phi mesons can be reconstructed via invariant mass technique applied to $K^{+} K^{-}$pairs. Due to the rescattering of the $K^{+}$and $K^{-}$ in the medium, the reconstructed invariant mass could result outside the original phi meson peak in such a way that some $\phi \rightarrow K^{+} K^{-}$events contribute to the experimental background rather than to the measured yields [7. The modification of the visible $\phi$ spectra due to kaon rescattering was first investigated within a basic RQMD model, showing that kaon rescattering can account for the observed rapidity distributions, but not for the differences in the $m_{t}$ spectra, neither the inverse slopes nor the relative yields, with a resulting suppression of the $\phi$ meson yield via the hadronic $K \bar{K}$ channel of $40-60 \%$ [7]. This effect has been further studied in the framework of a spherically expanding fireball model by including different possible changes (increasing/decreasing) of kaon masses in the hadronic medium [8], as well as in the framework of a multi-phase transport (AMPT) model by including density dependent in-medium modifications of kaons and $\phi$ mesons 9]. In none of these cases the large difference between the two SPS experiments could be explained. Such a large enhancement of four at low transverse mass from dimuon over the dikaon channel could only be explained in the AMPT model by an ad hoc increase of the phi meson number from the initial stage by about a factor of three, that might then suggest that other mechanisms, such as formation of colour ropes or quark-gluon plasma, are needed for $\phi$ meson production [9].

To shed light on this so called " $\phi$ puzzle", two experimental collaborations planned to measure the $\phi$ yield simultaneously in both $\phi \rightarrow e^{+} e^{-}$and $K \bar{K}$ channels at two different energy regimes, namely the CERES Collaboration at CERN SPS and the PHENIX Collaboration at the BNL Relativistic Heavy Ion Collider (RHIC). The CERES Collaboration measured $\phi$ meson production in central $\mathrm{Pb}+\mathrm{Au}$ collisions at $E_{l a b}=158 \mathrm{~A} \mathrm{GeV}$. Recently 
E. Santini et al.: Medium effects on $\phi$ decays to dilepton and kaon-antikaon pairs ...

reported data show that the yields and inverse slope parameters in both decay modes agree within errors. A yield in the $e^{+} e^{-}$decay mode larger than 1.6 times the yield in the $K^{+} K^{-}$channel is excluded at 95\% CL 10. The PHENIX Collaboration measured the $\phi$ yield in $\mathrm{Au}+\mathrm{Au}$ and $\mathrm{d}+\mathrm{Au}$ collisions at $\sqrt{s_{\mathrm{NN}}}=200 \mathrm{GeV}$ [1, 12,13,14. Whereas definitive results on the $\phi$ yield at mid-rapidity in the $\mathrm{Au}+\mathrm{Au}$ collisions as measured in the $K^{+} K^{-}$channel have been already reported 12 , only preliminary results on the corresponding dilepton channel are available so far 11,13 14. According to recently reported preliminary production results, the temperatures measured in the two decay channels are in agreement within errors, while the yield in the $e^{+} e^{-}$channel seems to be larger than in the $K^{+} K^{-}$decay channel. However, the errors in the dielectron channel, both statistical and systematic, are still too large for a definite statement [14. On the other side, recently reported preliminary data on the yields of both $\phi \rightarrow K^{+} K^{-}$and $\phi \rightarrow e^{+} e^{-}$in $\mathrm{d}+\mathrm{Au}$ collision show that the two data samples are consistent in both the total invariant yield and inverse slope [13].

In heavy ion reactions at energies reached at RHIC, the medium after hadronization is baryon dilute but meson rich [15. Since by far the most abundant particles are pions, one can speak about pion matter created in heavy ion collisions at RHIC energies. The kaon-pion gas was studied in detail within chiral perturbation theory (ChPT) in ref. 16, focusing thereby on the quark condensates. ChPT, proposed for the description of interactions of pseudo-scalar mesons at low energies, although being an adequate tool for studying the problem at low temperatures $\left(T \lesssim M_{\pi}\right)$, cannot be applied at typical temperatures reached at RHIC $(T \sim 200 \mathrm{MeV})$. At high temperatures, more phenomenological approaches come into play and ChPT provides a useful tool to control the low temperature limit of such phenomenological models.

In ref. [17, the modifications of kaon properties in isotopically symmetric hot pion matter has been calculated. Starting from kaon in-medium masses and mean field potentials calculated in isotopically symmetric pion matter to one loop of chiral perturbation theory, the results have been extended to RHIC temperatures using experimental data on $\pi K$ scattering phase shifts. Kaon in-medium broadening and $\phi$ in-medium width were calculated as well. Here we are using the results of ref. 17 to investigate the role of the rescattering of secondary kaons inside the pion matter on the apparent dilepton branching ratio of the $\phi$ meson.

The paper is organised as follows. In section 2 we briefly review the main results of kaon in-medium masses and broadening, mean field potential, as well as of $\phi$ meson in-medium width in an isotopically symmetric hot gas of pions investigated in ref. [17. In section 3 we discuss the identification of $\phi$ mesons from both the dimuon and dikaon channels and calculate its apparent dilepton branching ratio in the framework of an evolutionary Bjorken scenario for the expanding fireball. Summary and conclusions are finally given in section 4

\section{In-medium modification of kaons in pion matter}

We start with the $\phi$ meson in-medium width, which can be written in the form

$$
\Gamma_{\phi \rightarrow K \bar{K}}^{*}=\eta \Gamma_{\phi \rightarrow K \bar{K}}^{\mathrm{vac}}
$$

where the $\eta$ function

$$
\begin{aligned}
\eta= & \frac{1}{\pi^{2}} \int \frac{p^{* 3}\left(M_{\phi}^{*}, m_{1}^{*}, m_{2}^{*}\right)}{p^{* 3}\left(M_{\phi}, M_{K}, M_{K}\right)} \frac{M_{K}^{*} \Gamma_{K}^{*} d m_{1}^{* 2}}{\left(m_{1}^{* 2}-M_{K}^{* 2}\right)^{2}+\left(M_{K}^{*} \Gamma_{K}^{*}\right)^{2}} \\
& \times \frac{M_{K}^{*} \Gamma_{K}^{*} d m_{2}^{* 2}}{\left(m_{2}^{* 2}-M_{K}^{* 2}\right)^{2}+\left(M_{K}^{*} \Gamma_{K}^{*}\right)^{2}}
\end{aligned}
$$

contains the in-medium changes of the $\phi$ meson decay width. Here $p^{* 3}\left(M_{\phi}, M_{K}, M_{K}\right)$ and $p^{* 3}\left(M_{\phi}^{*}, M_{K}^{*}, M_{K}^{*}\right)$ are the kaon three-momenta in the $\phi$ meson rest frame for an in-vacuum and in-medium decay respectively. $\Gamma_{K}^{*}$ is the kaon collision width. The in-medium masses are $M_{\phi}^{*}=$ $M_{\phi}-2 V_{K}$ and $M_{K}^{*}=M_{K}+\delta M_{K}$, where $V_{K}$ is the mean field potential and $\delta M_{K}$ is the mass shift for kaons in isotopically symmetric pion matter [17.

The kaon collision width is given by

$$
\begin{aligned}
\Gamma_{K}^{*}= & \frac{1}{6 M_{K}(2 \pi)^{2}} \sum_{\ell} \int\left[\left|A_{\ell}^{1 / 2}(s)\right|^{2}+2\left|A_{\ell}^{3 / 2}(s)\right|^{2}\right] d n_{s \pi} \\
& \times \Phi_{2}\left(\sqrt{s}, M_{\pi}, M_{K}\right)
\end{aligned}
$$

where $A_{\ell}^{I}(s)$ are the on-shell partial wave projections of the $\pi K$ amplitudes with total isospin $I=1 / 2$ and $3 / 2$. The function $\Phi_{2}\left(\sqrt{s}, M_{\pi}, M_{K}\right)=\pi p^{* 3}\left(\sqrt{s}, M_{\pi}, M_{K}\right) / \sqrt{s}$ is the invariant $\pi K$ phase space with the c.m. momentum of the $\pi K$ system $p^{*}\left(\sqrt{s}, M_{\pi}, M_{K}\right)$. The scalar pion density is defined by $d n_{s \pi}=d n_{v \pi} /\left(2 E_{\pi}\right)$ via the number densities of pions given by the Bose distribution function

$$
d n_{v \pi}=\frac{d^{3} p_{\pi}}{(2 \pi)^{3}}\left[\exp \left(\frac{E_{\pi}-\mu_{\pi}}{T}\right)-1\right]^{-1}
$$

where $\mu_{\pi^{+}}=-\mu_{\pi^{-}}$is the $\pi^{+}$chemical potential and $\mu_{\pi^{0}}=0$.

The on-shell $\pi K$ amplitudes have been calculated in ChPT to the order $p^{4}$ by several authors (see e.g. [18] and references therein). To lowest order $p^{2}$, the off-shell $\pi K$ amplitudes are given in ref. 19. The self energy operator $\Sigma\left(M_{K}^{2}, M_{K}\right)$, mass-shift $\delta M_{K}$ and mean field potential $V_{K}$ for kaons in an isotopically symmetric pion gas have been calculated to leading order in pion density in chiral perturbation theory from the $\pi K$ forward scattering amplitudes determined at the one loop level. The amplitudes were expressed in terms of the $s$ - and $p$-wave $\pi K$ scattering lengths and the $s$-wave effective ranges. The derived expressions, valid at $T \lesssim M_{\pi}$, can be extended via a more phenomenological approach to typical chemical freeze-out temperatures at RHIC $(T \sim 170 \mathrm{MeV} 20$ ] by rewriting the $s$-wave parts of the amplitudes $A^{I}$ in terms of phase shifts and by assuming the behaviour of the $p$-wave to be fixed by the $a_{1}^{I}$ scattering length and 

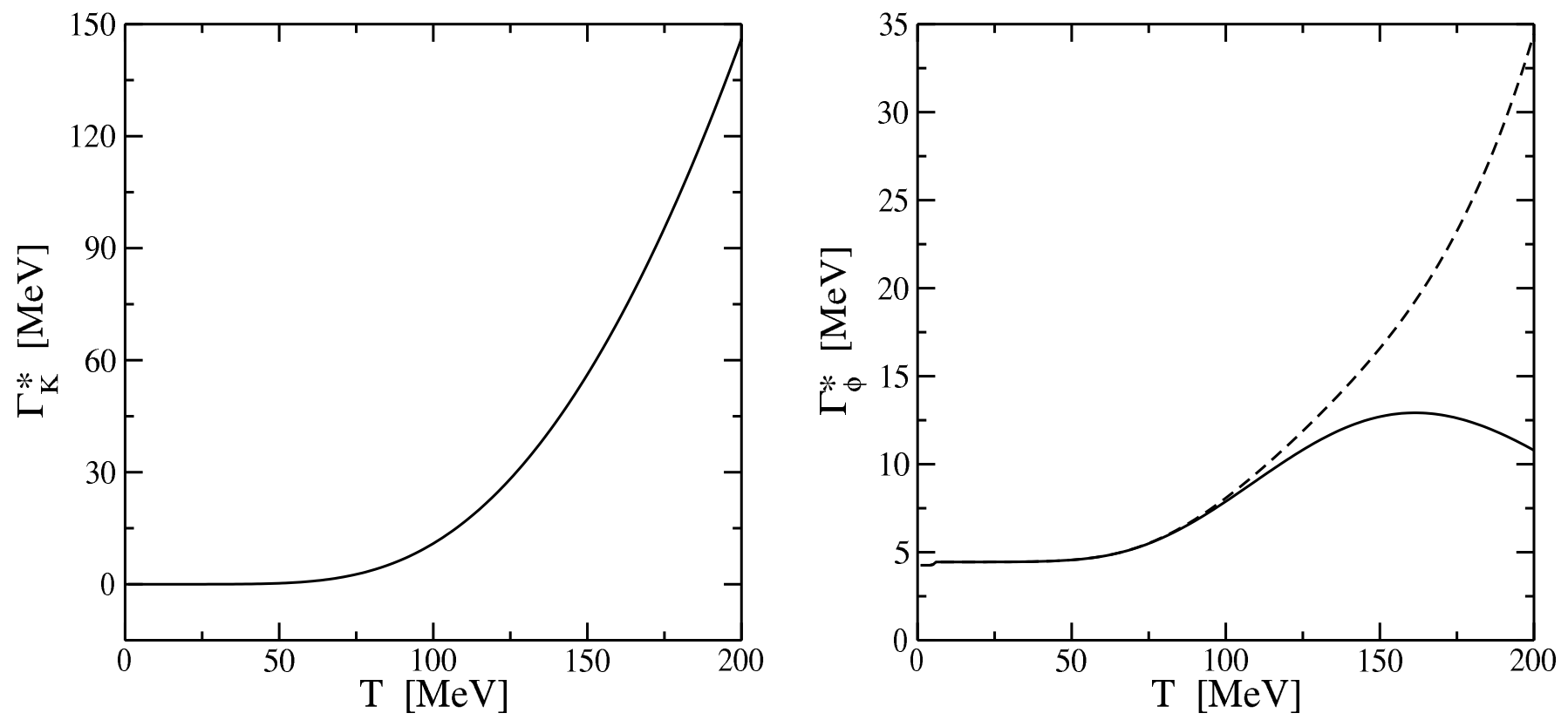

Fig. 1. Kaon collision width $\Gamma_{K}^{*}$ (left) and in-medium $\phi$ meson width $\Gamma_{\phi \rightarrow K \bar{K}}^{*}$ (right, solid line) versus temperature $T$ in isotopically symmetric pion matter. The dashed curve in the right panel corresponds to a phenomenologically increased $\phi$ width $\Gamma_{\phi}^{t o t}=\Gamma_{\phi}^{*}+\Gamma_{\phi+\rho}^{c o l l}$, in which an additional collisional broadening by $\rho$ mesons is taken into account (see the detailed discussion in section 3 .

the resonance $K^{*}$. The resulting amplitudes satisfy unitarity. The experimental $\pi K$ scattering phases are then well reproduced; the low-temperature limit matches smoothly with one-loop ChPT. For the details, we refer to ref. [17. The resulting kaon collision width and in-medium $\phi$ meson width are shown in fig. Mas a function of temperature in the left and right panel respectively.

\section{Apparent dilepton branching ratio of the $\phi$ meson}

Since the $\phi$ meson is unstable, it can only be detected from its decay products of either the kaon-antikaon pair or the lepton pair. Dileptons from $\phi$ decays leave the pion matter essentially undistorted by final state interactions, whereas the secondary kaons rescatter with the medium and can contribute to the experimental background. We investigate whether this can result in an increase of the apparent dilepton branching ratio or not.

Let us assume that $\phi$ mesons leave the heavy ion reaction at time $\tau$. In the simple case of constant widths, as already pointed out in ref. 17, the probability for the kaons to leave the reaction zone without rescattering is given by

$$
w \sim \int_{0}^{\tau} \exp \left(-2 \Gamma_{K}^{*}(\tau-t)\right) \exp \left(-\Gamma_{\phi}^{*} t\right) \Gamma_{\phi} d t
$$

The first factor in this expression is the probability for two kaons to escape from the reaction zone without rescattering. The second factor is the survival probability of the $\phi$ meson at time $t$. The last factor $\Gamma_{\phi} d t$ is the probability to decay into the kaon pair during $d t$. Notice that $\Gamma_{\phi}^{*} d t$ has the meaning of a decay probability into the kaons which rescatter with pions from the surrounding medium. Selecting kaon pairs with invariant masses of the $\phi$ meson which suffered no rescatterings, the number of $\phi$ mesons observed in the $K \bar{K}$ channel equals

$$
\begin{aligned}
N_{K \bar{K}} \sim \exp \left(-\Gamma_{\phi}^{*} \tau\right)+ & \frac{\Gamma_{\phi}}{2 \Gamma_{K}^{*}-\Gamma_{\phi}^{*}}\left[\exp \left(-\Gamma_{\phi}^{*} \tau\right)\right. \\
& \left.-\exp \left(-2 \Gamma_{K}^{*} \tau\right)\right] .
\end{aligned}
$$

The first term arises due to the vacuum decays, whereas the second term is given by eq. (5). The number of $\phi$ mesons observed in the dilepton channel equals

$$
N_{e^{+} e^{-}} \sim B \exp \left(-\Gamma_{\phi}^{*} \tau\right)+B^{*}\left[1-\exp \left(-\Gamma_{\phi}^{*} \tau\right)\right],
$$

where $B$ and $B^{*}$ are the vacuum and in-medium dilepton branching ratios of the $\phi$ meson respectively. We assume that the dilepton channel suffers no in-medium modifications, i.e. $B^{*} / B=\Gamma_{\phi} / \Gamma_{\phi}^{*}$. Notice that the second term of eq. (77) can be also written as

$$
\begin{aligned}
B^{*}\left[1-\exp \left(-\Gamma_{\phi}^{*} \tau\right)\right] & =\int_{0}^{\tau} \exp \left(-\Gamma_{\phi}^{*} t\right) \Gamma_{\phi \rightarrow e^{+} e^{-}} d t \\
& =\int_{0}^{\tau} \exp \left(-\Gamma_{\phi}^{*} t\right) B \Gamma_{\phi} d t,
\end{aligned}
$$

whose interpretation is straightforward, being $\exp \left(-\Gamma_{\phi}^{*} t\right)$ the survival probability of the $\phi$ meson at time $t$ and $\Gamma_{\phi \rightarrow e^{+} e^{-}} d t$ its probability to decay into a dilepton pair during $d t$. 
Turning now to temperature dependent, and therefore time dependent widths, the previous relations should be modified in the following way:

$$
\begin{aligned}
\exp \left(-2 \Gamma_{K}^{*}(\tau-t)\right) & \Longrightarrow \exp \left(-2 \int_{t}^{\tau} \Gamma_{K}^{*}\left(t^{\prime}\right) d t^{\prime}\right) \\
\exp \left(-\Gamma_{\phi}^{*} t\right) & \Longrightarrow \exp \left(-\int_{0}^{t} \Gamma_{\phi}^{*}\left(t^{\prime}\right) d t^{\prime}\right) \\
\exp \left(-\Gamma_{\phi}^{*} \tau\right) & \Longrightarrow \exp \left(-\int_{0}^{\tau} \Gamma_{\phi}^{*}\left(t^{\prime}\right) d t^{\prime}\right)
\end{aligned}
$$

so that the number of $\phi$ mesons decaying into $K \bar{K}$ pairs is determined by

$$
\begin{aligned}
N_{K \bar{K}} \sim & \exp \left(-\int_{0}^{\tau} \Gamma_{\phi}^{*}\left(t^{\prime}\right) d t^{\prime}\right)+\int_{0}^{\tau} \exp \left(-2 \int_{t}^{\tau} \Gamma_{K}^{*}\left(t^{\prime}\right) d t^{\prime}\right) \\
& \times \exp \left(-\int_{0}^{t} \Gamma_{\phi}^{*}\left(t^{\prime}\right) d t^{\prime}\right) \Gamma_{\phi} d t
\end{aligned}
$$

and those decaying into dileptons by

$$
\begin{aligned}
N_{e^{+} e^{-}} \sim & B \exp \left(-\int_{0}^{\tau} \Gamma_{\phi}^{*}\left(t^{\prime}\right) d t^{\prime}\right) \\
& +B \int_{0}^{\tau} \exp \left(-\int_{0}^{t} \Gamma_{\phi}^{*}\left(t^{\prime}\right) d t^{\prime}\right) \Gamma_{\phi} d t
\end{aligned}
$$

Finally, the apparent dilepton branching ratio with respect to the vacuum branching ratio is then simply given by the ratio of both quantities (9) and (10), i.e.

$$
\frac{B^{a p p}}{B}=\frac{N_{e^{+} e^{-}}}{N_{K \bar{K}}} \text {. }
$$

In central relativistic heavy ion collisions the distribution of matter is approximatively uniform in rapidity, at least in the mid-rapidity region, and the geometry of the collision can be considered as cylindrically symmetric [21. In the framework of a Bjorken scenario, extensively used to describe the space-time evolution of ultrarelativistic heavy ion collisions, the time and temperature of the evolving system are related according to [21] by

$$
\frac{T(t)}{T_{0}}=\left(\frac{t_{0}}{t}\right)^{c_{s}^{2}}
$$

where $c_{s}^{2}$ is the sound velocity squared for a baryon less gas, and $T_{0}$ is the initial temperature of the system that is assumed to be fully thermalized after the time $t_{0}$. Because our investigations are restricted to matter created in heavy ion reactions after hadronization, we have applied eq. (12) to describe the temporal evolution of the hadronized system reinterpreting $t_{0}$ and $T_{0}$ by the hadronization time $t_{h}$ and hadronization temperature $T_{h}$ respectively. For an ideal relativistic gas, the energy density and the pressure are related by $\epsilon=3 p$, therefore one finally has

$$
\frac{1}{c_{s}^{2}}=\frac{d \epsilon}{d p}=3 \quad \text { and } \quad \frac{T(t)}{T_{h}}=\left(\frac{t}{t_{h}}\right)^{-1 / 3} .
$$

In section 2 expressions for the kaon collision width and in-medium $\phi$ meson width as functions of the temperature were given. The apparent dilepton branching ratio can be evaluated from eq. (11) using eqs. (9) and (10) by performing the $t \rightarrow T$ variable replacement according to the transformation law (13). In addition to the onset of the $T(t)$ evolution, the parameters $T_{h}$ and in particular $t_{h}$ determine mainly the slope of the temperature decrease with ongoing expansion. Thus, both parameters may strongly influence the final results in the following way: the larger $T_{h}$ and $t_{h}$ are, i.e. the slowlier the hadronic phase cools down, the stronger the in-medium effects are. The resulting ratios $B^{a p p} / B$ for three different sets of these parameters are shown in fig. 2 as a function of the phi mesons's escape time $\tau$. First of all, we chose in our model cal-

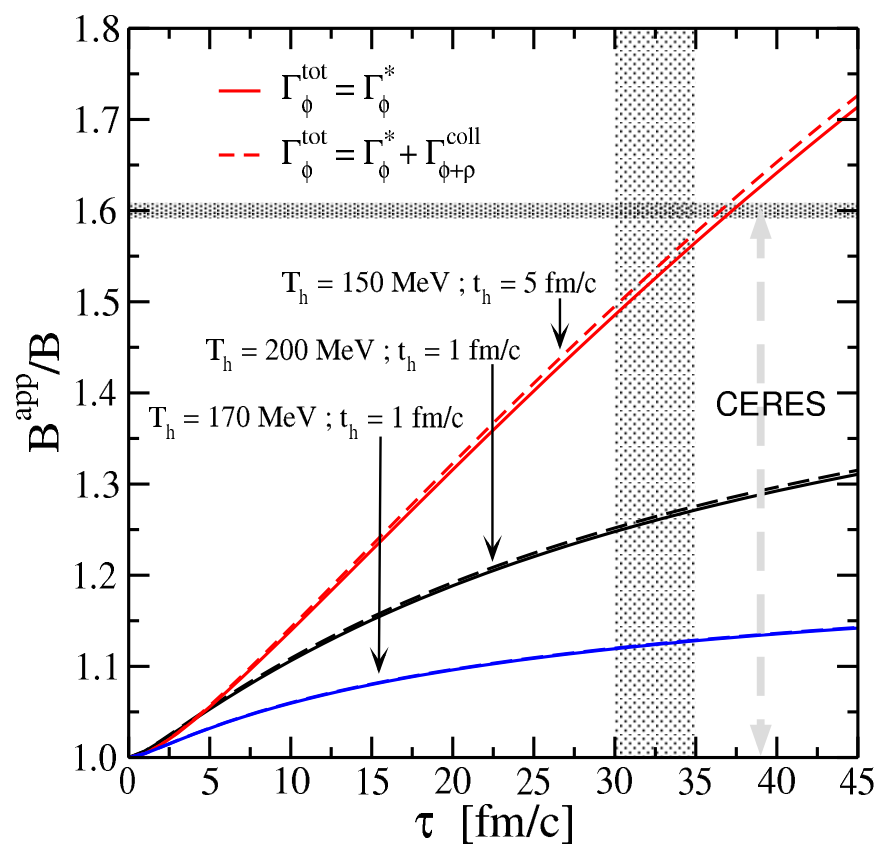

Fig. 2. Ratio between the apparent and vacuum dilepton branchings $B^{a p p} / B$ versus the $\phi$ meson's escape time $\tau$ (solid curves). The dashed curves correspond to the case in which an additional collisional broadening in the phi meson width is taken into account (see right panel of fig. 1). The vertically hatched area corresponds to a region around a $\tau$ value of $\approx 32 \mathrm{fm} / \mathrm{c}$ predicted by transport calculations for RHIC conditions. The horizontally hatched band at $B^{a p p} / B=1.6$ indicates the upper limit of the enhancement factor according to the observations of the CERES Collaboration at SPS (see discussion below).

culation an initial temperature of $T_{h}=200 \mathrm{MeV}$ and a corresponding time of $t_{h}=1 \mathrm{fm} / \mathrm{c}$ leading to a fast cooling behaviour of the expanding fireball. In this quasi extreme scenario, we find that the phi meson yield from the dilepton channel is only slightly higher by a factor of about 1.25 than that from the $K \bar{K}$ channel - at least in the physically interesting $\tau$ region. According to transport calculations, the term $\exp \left(-\tau \Gamma_{\phi}\right)$ is of the order $\sim 1 / 2$ at RHIC energies 22, i.e. $\tau \sim 32 \mathrm{fm} / \mathrm{c}$. Therefore, the ex- 
pected region of physical interest is depicted in fig. 2 as vertically hatched area. The $B^{a p p} / B$ ratio from a model calculation with practically the same fast cooling, but a smaller and therefore more realistic hadronization temperature of $T_{h}=170 \mathrm{MeV}$ according to transition temperatures obtained from 2-flavour lattice QCD [23] is even essentially smaller, because the interval of the temperature integration is shrinked. The consequences of a larger hadronization time are also depicted in fig. 2. With a value of $t_{h}=5 \mathrm{fm} / \mathrm{c}$, the temperature of the expanding hadronic fireball decreases much slowlier compared to the previous parametrizations. In this case, the in-medium effects influence the ratio between the apparent and the vacuum dilepton branchings $B^{a p p} / B$ in a much stronger manner, that overcompensates the (converse) effect of an even smaller hadronization temperature $T_{h}=150 \mathrm{MeV}$ according to transition temperatures obtained from 3-flavour lattice QCD 23. The enhancement factor in the latter scenario turns out to be about $1.5-1.6$ in the region around a $\tau$ value of $\approx 32 \mathrm{fm} / \mathrm{c}$.

Nevertheless, all these values are in qualitative agreement with the observations of the CERES Collaboration at SPS energies, who excludes an enhancement factor larger than 1.6 at $95 \% \mathrm{CL}^{1}$, and contrasts the strong enhancement factor of $2-4$ resulting from the comparison between the yields measured for the $\mu^{+} \mu^{-}$and $K^{+} K^{-}$channels by the NA50 and NA49 Collaborations respectively. Well, a large enhancement of that magnitude is still not totally excluded within the simplified view of a Bjorken scenario, that assumes only a longitudinally expanding medium. We want to emphasise once more that the question, how fast the temperature of the hadronic fireball decreases, seems to be very crucial. A more realistic description taking additionally a transversal expansion into account might promote a fast cooling scenario, that tends to result in not so strong enhancement factors. The exclusion of a large enhancement due to in-medium effects and kaon collisional broadening in heavy ion collisions at RHIC energies results also from multi-phase transport calculations. In particular, the production of $\phi$ mesons reconstructed from dikaon and dilepton decays has been investigated in ref. 9] for central $\mathrm{Au}+\mathrm{Au}$ collisions at a RHIC energy of $\sqrt{s_{\mathrm{NN}}}=130 \mathrm{GeV}$. There have been included density dependent in-medium modifications of kaon masses derived from a chiral effective Lagrangian and a linear decrease of the $\phi$ mass according to a scaling law $m_{\phi}^{*} \approx m_{\phi}\left(1-\alpha \rho / \rho_{0}\right)$, with $\alpha=0.0255$ and the normal nuclear matter density $\rho_{0}$. It was found that the $\phi$ meson yield from the dilepton channel is about 1.5 times larger than that from the $K^{+} K^{-}$channel in the absence of additional $\phi$ meson production due to the formation of an initial partonic stage.

The idea of a $\phi$ meson which decouples easily from the hadronic fireball is widely diffused, as a consequence of the small cross section for its scattering with non-strange hadrons. However, available calculations give contradictory results $24,25,26,27]$. Whereas some of them support

1 The $\phi$ yields in the leptonic and the hadronic channel published in ref. 10 result in a ratio $B^{a p p} / B$ of $1.0 \pm 0.31$ (stat) \pm 0.28 (syst), i.e. a maximum value of about 1.6 (upper limit). this idea [24 25 26], a calculation based on a Hidden Local Symmetry Lagrangian shows that the collision rates of the $\phi$ meson in a hot hadronic matter of pseudo-scalar $(\pi$, $K)$ and vector mesons $\left(\rho, \omega, K^{*}, \phi\right)$ is rather large 27 and dominated at temperatures between $150 \mathrm{MeV}$ and 200 $\mathrm{MeV}$ by the $K^{*}$ followed by $K$ and $\rho$ contributions, while the contributions from $\pi, \omega$ and $\phi$ are smaller. The corresponding $\phi$ mean free path at temperatures above 170 $\mathrm{MeV}$ is between $2.4 \mathrm{fm}$ and $1 \mathrm{fm}$, and therefore considerably smaller than the typical size of the hadronic system created in heavy ion collisions $(\sim 10-15 \mathrm{fm})$. This would imply that most $\phi$ mesons produced after the hadronization would not leave the hadronic system without scattering. It was found, that reactions changing the number of $\phi$ mesons account for more than $80 \%$ of the total collision rate, so that at the early stage of the expansion, when the effect of the decay is negligible, the collision rate is mainly responsible for the decrease of the $\phi$ number with respect to its value at the hadronization.

Since a pion gas is only the first approximation to the hadronic final state of a relativistic heavy ion collision one can estimate the stability of the present investigations by the inclusion of the $\phi+\rho \rightarrow K \bar{K}$ channel, i.e. the next important channel which gives - according to the results in ref. 27 - rise to in-medium modifications of the $\phi \rightarrow K \bar{K}$ decay. However, in contrast to the influence of the pion gas 17. we consider the contributions from the $\rho$ mesons in a phenomenological way: following ref. 27] the collisional width $\Gamma_{\phi+\rho}^{c o l l}$ due to the scattering of $\phi$ 's on $\rho$ mesons is added to the in-medium width $\Gamma_{\phi}^{*}$ from the interaction with the pions. To do so we extrapolated the results of ref. 27] imposing the boundary condition $\Gamma_{\phi+\rho}^{c o l l}=0$ at $T=0$ as well as a fast decrease to zero for $T \rightarrow 0$ and calculated the apparent dilepton branching ratio using this time a total $\phi$ meson width equal to $\Gamma_{\phi}^{\text {tot }}=\Gamma_{\phi}^{*}+\Gamma_{\phi+\rho}^{c o l l}$. Fig. 11 shows the obtained total $\phi$ meson width $\Gamma_{\phi}^{\text {tot }}$ as a function of temperature. Obviously in a very high but narrow temperature range, the $\phi+\rho$ collision rate dominates on the in-medium $\phi$ decay width. When the system cools down, the additional rate drops fast and becomes negligible compared to $\Gamma_{\phi}^{*}$ in the lower temperature domain.

Fig. 2 depicts also the corresponding ratios $B^{a p p} / B$ with comparison to their values obtained when the collisional broadening due to $\rho$ mesons is neglected. As one can easily see, the introduction of a collisional broadening does not significantly influence the apparent dilepton branching ratio. The increase of the total $\phi$ width reduces simultaneously the phi decays into dileptons as well as the number of $\phi$ mesons reconstructed via the $K \bar{K}$ decay channel. The two reduction factors turn out to be comparable in magnitude, so that at the end the ratio $B^{a p p} / B$ is only very slightly additionally enhanced. This fact is retained for all of the three parametrizations being under consideration. Furthermore, this fact demonstrates that for the present purpose the fireball expansion after hadronization can safely be described by an expanding pion gas. The reason is simply that the integration over the entire space-time volume of the expanding system is 
E. Santini et al.: Medium effects on $\phi$ decays to dilepton and kaon-antikaon pairs ...

dominated by the lower temperatures where the heavier mesons are suppressed by their Boltzmann factors.

\section{Summary and conclusions}

We have investigated the role of the rescattering of secondary kaons inside pionic matter on the apparent dilepton branching ratio of the $\phi$ meson in heavy ion collisions at RHIC energies, taking into account in-medium mass modifications and broadening of kaons and $\phi$ mesons in an isotopically symmetric hot pion gas. We have found within the framework of a Bjorken scenario for the temporal evolution of the expanding hadronic fireball that the phi meson yield reconstructed from the dilepton channel is only by a factor between about 1.1 and 1.6 higher than that reconstructed from the kaon-antikaon channel. We have demonstrated that the magnitude of this enhancement depends also on the question, how slowly the temperature of the pionic medium decreases. Nevertheless, for reasonable parametrizations of the cooling scenario, our results agree quantitatively well with recent observations of the CERES Collaboration at CERN SPS energies. The inclusion of further in-medium effects through the addition of a possible collisional broadening in the $\phi$ meson in-medium width does not change considerably our results. For heavy ion collisions at RHIC energies, only preliminary results on the $\phi$ meson yield measured via the two decay channels are available up to now. The errors in the dilepton channel are unfortunately still too large for a definitive statement. Future data from RHIC will help bring more insight into the meson propagation in a dense and hot medium.

\section{Acknowledgements}

This work was supported by the European Graduate School Basel-Graz-Tübingen and by the Bundesministerium für Bildung und Forschung (BMBF) under contract 06TÜ202.

\section{References}

1. J. Rafelski and B. Müller, Phys. Rev. Lett. 48, 1066 (1982); [Erratum-ibid. 56, 2334 (1986)];

P. Koch, B. Müller and J. Rafelski, Phys. Rep. 142, 167 (1986).

2. A. Shor, Phys. Rev. Lett. 54, 1122 (1985).

3. S. Okubo, Phys. Lett. 5, 165 (1963);

G. Zweig, CERN Report No.8419/TH, 412 (1964);

J. Iizuka, Prog. Theor. Phys. Suppl. 37, 21 (1966).

4. C. Höhne et al. [NA49 Collaboration], Nucl. Phys. A 661, 485 (1999);

S. V. Afanasev et al. [NA49 Collaboration], Phys. Lett. B 491, 59 (2000).

5. M. C. Abreu et al. [NA50 Collaboration], Nucl. Phys. A 661, 543 (1999);

B. Alessandro et al. [NA50 Collaboration], Phys. Lett. B 555, 147 (2003); [Erratum-ibid. B 561, 294 (2003)].

6. D. Röhrich, J. Phys. G 27, 355 (2001).
7. S. C. Johnson, B. V. Jacak and A. Drees, Eur. Phys. J. C 18, 645 (2001).

8. P. Filip and E. E. Kolomeitsev, Phys. Rev. C 64, 054905 (2001).

9. S. Pal, C. M. Ko and Z. Lin, Nucl. Phys. A 707, 525 (2002).

10. D. Adamova et al. [CERES Collaboration], Phys. Rev. Lett. 96, 152301 (2006).

11. J. L. Nagle [PHENIX Collaboration], Nucl. Phys. A 715, 252 (2003).

12. S. S. Adler et al. [PHENIX Collaboration], Phys. Rev. C 72, 014903 (2005).

13. K. Ozawa et al. [PHENIX Collaboration], Eur. Phys. J. C 43, 421 (2005).

14. A. Kozlov et al. [PHENIX Collaboration], [arXiv: nucl-ex/0510016.

15. K. Adcox et al. [PHENIX Collaboration], Phys. Rev. Lett. 86, 3500 (2001);

B. B. Back et al. [PHOBOS Collaboration], Phys. Rev. C 65, 031901 (2002).

16. A. Dobado and J. R. Peláez, Phys. Rev. D 59, 034004 (1999).

17. B. V. Martemyanov, Amand Faessler, C. Fuchs and M. I. Krivoruchenko, Phys. Rev. Lett. 93, 052301 (2004).

18. A. G. Nicola and J. R. Peláez, Phys. Rev. D 65, 054009 (2002).

19. J. A. Cronin, Phys. Rev. 161, 1483 (1967);

R. W. Griffith, Phys. Rev. 176, 1705 (1968).

20. P. Braun-Munzinger et al., Phys. Lett. B 518, 41 (2001).

21. J. D. Bjorken, Phys. Rev. D 27, 140 (1983).

22. L. Bravina et al., Nucl. Phys. A 715, 665 (2003).

23. F. Karsch and E. Laermann, in: R. C. . Hwa (ed.) and X. N. . Wang (ed.), Quark-gluon plasma. Vol. 3, 1 (2004); arXiv:hep-lat/0305025.

24. C. M. Ko and D. Seibert, Phys. Rev. C 49, 2198 (1994).

25. K. Haglin, Nucl. Phys. A 584, 719 (1995).

26. W. Smith and K. L. Haglin, Phys. Rev. C 57, 1449 (1998).

27. L. Alvarez-Ruso and V. Koch, Phys. Rev. C 65, 054901 (2002). 